\title{
New Definitions of the Kilogram and the Mole: Paradigm Shift to the Definitions Based on Physical Constants
}

\author{
Naoki KuRAMoto \\ National Metrology Institute of Japan, National Institute of Advanced Industrial Science and Technology, \\ 1-1-1 Umezono, Tsukuba, Ibaraki 305-8563, Japan
}

\begin{abstract}
The kilogram is the unit of mass and was defined in 1889 by the international prototype of the kilogram. The mole is the unit of amount of substance and was defined in 1960 by the number of atoms in $0.012 \mathrm{~kg}$ of ${ }^{12} \mathrm{C}$. These definitions were revised in May 2019. The new definitions of the kilogram and the mole are based on the Planck constant $h$ and the Avogadro constant $N_{\mathrm{A}}$, respectively. The values of $h$ and $N_{\mathrm{A}}$ used in the new definitions were determined by summarizing measurement results of the two physical constants by several national metrology institutes around the world. In this review, the history of the two units and measurement technologies used to derive the new definitions are described. The effect of the revision on the development of new measurement technologies is also introduced.
\end{abstract}

Keywords Kilogram, mole, Planck constant, Avogadro constant, new SI

(Received August 29, 2020; Accepted November 13, 2020; Advance Publication Released Online by J-STAGE November 20, 2020)

1 Introduction

2 Kilogram and Mole

3 Recommendation to Retire the International

Prototype of the Kilogram

4 Kibble Balance

5 X-ray Crystal Density Method

$5 \cdot 1{ }^{28} \mathrm{Si}$-enriched crystal

$5 \cdot 2$ Sphere volume measurement

5.3 Sphere mass measurement

$5 \cdot 4$ Surface characterization

$5 \cdot 5$ Volume and mass of Si core

5.6 Lattice constant measurement

5.7 Molar mass measurement

5.8 Avogadro constant and Planck constant
177

178

178

179

179
6 Determination of the Planck Constant in the New Definition of the Kilogram

7 New Definition of the Mole Based on the Avogadro Constant

8 Impact of the Redefinition of the Kilogram

8.1 Dissemination of the kilogram under the new definition

8.2 Small mass measurement based on the planck constant

9 Impact of the Redefinition of the Mole

10 Impact of the Redefinition on New Science and Technology

11 Acknowledgements

12 References
185

186

186

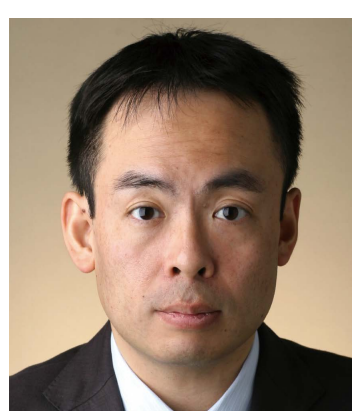

Naoki Kuramoto is the Group Leader of the Mass Standards Group of the National Metrology Institute of Japan/National Institute of Advanced Industrial Science and Technology (NMIJ/AIST). He received his Ph.D. degree in chemistry from Saga University in 1998. Since 1999, he has been working at NMIJ/ AIST, where he developed an optical interferometer to measure the volume of ${ }^{28} \mathrm{Si}$-enriched spheres for the redefinition of the kilogram. The Planck constant determined by Kuramoto et al. in 2017 had a significant impact on the determination of the Planck constant in the new definition of the kilogram. His research interests include development of new mass measurement principles based on the Planck constant. He also serves as the Coordinator of the International Avogadro Coordination project.

E-mail: n.kuramoto@aist.go.jp

\section{Introduction}

Measurement is one of the most important fundamental elements of natural science, and human beings have been trying to understand various phenomena of this world by developing diverse measurement technologies. To investigate unknown phenomena in cooperation with researchers around the world, common measurement standards are essential. Currently, the International System of Units $(\mathrm{SI})^{1}$ provides the standards. Furthermore, in order to realize more accurate measurement standards, the most advanced technologies in each era have been used for the definitions of various units in the SI. That is, the definitions of units are not eternal and continue to evolve with the development of science and technology.

The kilogram with the symbol $\mathrm{kg}$ and the mole with the symbol mol are the SI units of mass and amount of substance, 


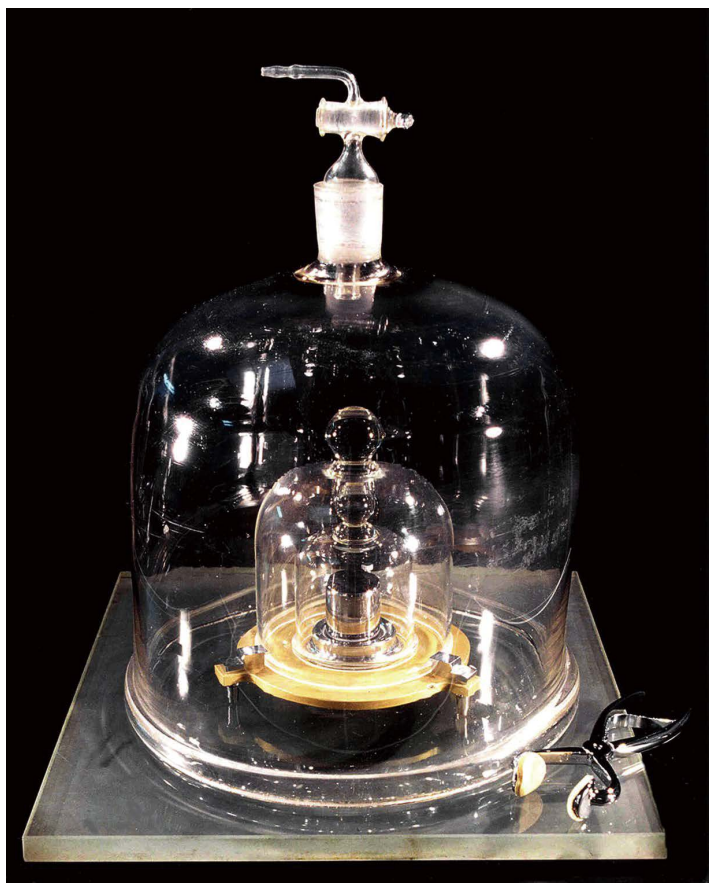

Fig. 1 International prototype of the kilogram enclosed by three bell jars: This cylindrical weight made of a platinum-iridium alloy is kept at the Bureau International des Poids et Mesures (BIPM) in Sèrves, France. The diameter and height are both approximately $39 \mathrm{~mm}$ (Photograph courtesy of the BIPM).

respectively. ${ }^{1}$ In May 2019, the SI was essentially revised. In the new SI, the kilogram is defined by fixing the value of the Planck constant, and the mole is defined by fixing the value of the Avogadro constant. In this review, the history of the two units and measurement technologies used to derive the new definitions are described. The impact of the revision on the development of new measurement technologies is also introduced.

\section{Kilogram and Mole}

The origin of the kilogram dates back to France toward the end of the 18th century. ${ }^{2}$ During the French Revolution, Lavoisier et al. measured the density of water, and the kilogram was defined as the mass of 1 liter of water at its melting point. This definition was then materialized by a platinum cylindrical weight. This weight is known as the "kilogram of archives" because it was stored in the National Archives of France in 1799. The mass of this weight was equal to the mass of 1 liter of water within the accuracy of measurement technologies at that time. The kilogram of archives was used as the standard for mass measurements until 1889.

In the 1870 s, the time was ripe to develop a new international standard of mass, taking advantage of the latest technologies. ${ }^{3}$ The material chosen for the new standard was an alloy of $90 \%$ platinum and $10 \%$ iridium, which is harder and less likely to wear than pure platinum. A cylindrical weight made of this alloy known as the international prototype of the kilogram was sanctioned as the artifact whose mass defines the kilogram in 1889 and was stored at the Bureau International des Poids et Mesures (BIPM, France) (Fig. 1). Surprisingly, the same weight was continuously used to define the kilogram for 130 years until
2019.

On the other hand, amount of substance is the quantity that specifies the amounts of chemical elements and compounds. The mole is the unit of amount of substance in the SI sanctioned in 1971. ${ }^{4}$ Before 2019, the mole was defined as the amount of substance of a system that contains as many elementary entities as there are atoms in $0.012 \mathrm{~kg}$ of ${ }^{12} \mathrm{C}$. The number of elementary particles contained in $1 \mathrm{~mol}$ was called the Avogadro constant, which was about $6.02 \times 10^{23} \mathrm{~mol}^{-1}$. The previous definition of the mole was therefore directly linked with the definition of the kilogram.

\section{Recommendation to Retire the International Prototype of the Kilogram}

The kilogram and the mole are the base units in the SI together with the second, the meter, the ampere, the kelvin, and the candela. ${ }^{1}$ As a result of pursuing high universality and reproducibility, the definitions of the base units were changed to those based on physical constants or properties peculiar to substances. For example, the meter was defined as the length of the international prototype of the meter in $1889 .{ }^{3}$ However, this definition was replaced in 1960 by one based on the wavelength of the radiation corresponding to a transition in ${ }^{86} \mathrm{Kr}$. In turn, the meter was redefined in 1983 as the distance traveled by light in a vacuum in 1/299 $792458 \mathrm{~s}$. The role of the international prototype of the meter was therefore taken over by the speed of light in vacuum, which is constant anywhere around the world and does not change over time. With appropriate technology, it is therefore possible to create a standard for length measurement without relying on special artifacts such as the international prototype of the meter.

On the other hand, the kilogram was continued to be defined by the international protype of the kilogram until 2019 . However, the international prototype of the kilogram is an artifact and its mass could change. For example, the international prototype of the kilogram was stored with its copies made of the same material. Since 1889 the mass differences between the international prototype of the kilogram and the copies drifted by about $50 \mu \mathrm{g}$ on the average..$^{5,6}$ A possible stability of the mass of the international prototype of the kilogram over 100 years was therefore estimated to be several tens of $\mu \mathrm{g}$. This corresponds to a relative mass change of the order of $10^{-8}$, which could not be ignored as measurement technology advances. Several national metrology institutes around the world therefore attempted to measure one of physical constants with a relative uncertainty of the order of $10^{-8}$ or smaller to define the kilogram using its value. On the basis of the research results, a policy to abolish the international prototype of the kilogram and move to the definition based on the Planck constant in the future was decided at the Conférence Générale des Poids et Mesures (CGPM) held in 2011.?

The Planck constant is a physical constant that describes various phenomena in the microscopic world. For example, it is related to the mass of an atom and the energy of a photon; $1 \mathrm{~kg}$ can therefore be expressed by the mass of a certain number of atoms or the mass of a body whose equivalent energy is equal to that of a certain number of photons. The Planck constant was measured in two ways, the Kibble balance ${ }^{8}$ and the $\mathrm{x}$-ray crystal density (XRCD) method. ${ }^{9}$ In 2013, the Consultative Committee for Mass and Related Quantities (CCM) of the Comite International des Poids et Mesures (CIPM) set a number of conditions that should be met before the redefinition of the kilogram. ${ }^{10}$ One of the conditions required that at least three 


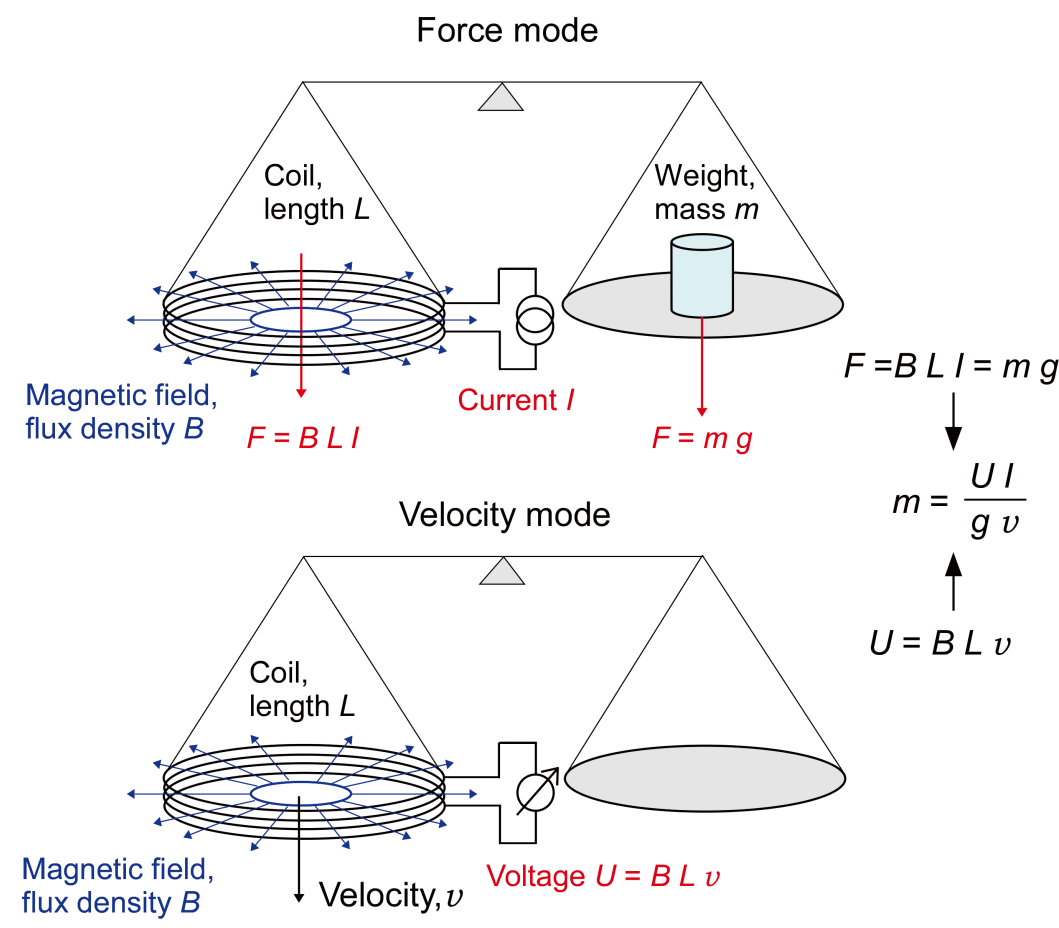

Fig. 2 Principle of the Kibble balance. Top: Force mode. The downward electromagnetic force generated by the coil $F$ is balanced with the gravitational force exerted on the weight $m g$. Bottom: Velocity mode. The coil moves vertically with a velocity of $v$ in a radial magnetic field, and a voltage $U$ is induced.

independent experiments using the Kibble balance and the XRCD method yield the values of the Planck constant with relative standard uncertainties not larger than $5 \times 10^{-8}$. In addition, at least one of these results should have a relative uncertainty not larger than $2 \times 10^{-8}$. Many national metrology institutes around the world therefore attempted to measure the Planck constant to fulfill these conditions.

\section{Kibble Balance}

In the Kibble balance, the gravitational force on an object is balanced with an electromagnetic force generated by a currentcarrying coil immersed in a magnetic field. Figure 2 shows the measurement principle. The measurement involves two modes of operation: force mode and velocity mode. In the force mode, downward force $F$ is generated by applying electric current $I$ through a coil of length $L$ placed in a magnetic field of magnetic flux density $B$. The electric current $I$ is measured when the generated $F$ balances the gravitational force on the weight of mass $m$, where $F$ and $m$ are related to $I$ by

$$
F=B L I=m g,
$$

where $g$ is the gravitational acceleration. On the other hand, in the velocity mode, the coil is moved at a vertical velocity $v$ so that a voltage $U$ is induced. The induced voltage $U$ is measured and is related to the velocity $v$ through the flux integral $B L$ by

$$
U=B L v
$$

When the quantity $B L$ is unchanged between the force mode and the velocity mode, one obtains the following equation by summarizing the measured quantities in the two modes.

$$
U I=m g v
$$

For the measurements of $U$ and $I$, electrical standards based on two quantum physical effects, the Josephson effect and the quantum Hall effect, are used. The Planck constant is directly related to these two quantum effects. ${ }^{11}$ By measuring $m, g, v, U$, and $I$ based on mass, length, time and electrical standards, we can therefore determine the Planck constant. The equations in this section and Fig. 2 are simplified by neglecting the vector notation. The rigorous equations with the vector notation are described by Eichenberger et al. ${ }^{12}$

In principle, the Planck constant can be measured only by operating in the force mode, but this requires that $B$ and $L$ are known. Because both variables are difficult to measure accurately, the velocity mode is necessary as a calibration technique to cancel out the $B L$ factor in Eq. (1).

\section{X-ray Crystal Density Method}

The x-ray crystal density (XRCD) method leads to a determination of the Avogadro constant. ${ }^{9}$ Details of the Avogadro constant are described in Sect. 3, and this constant can be converted to a value of the Planck constant using a rigorous relationship between the two physical constants. ${ }^{10}$ The fundamental principle of the XRCD method is the counting of atoms in a single crystal. Silicon crystals are used as a sample material of this method, mainly because the semiconductor industry developed growth technologies for large silicon single crystals having extremely high chemical purity and no dislocations. Figure 3 shows the unit cell of silicon: a cube with edge length $a$, which contains eight silicon atoms. ${ }^{13}$ When the volume $V$ of a silicon crystal sample is measured, the number $N$ of silicon atoms in the crystal is therefore given by 


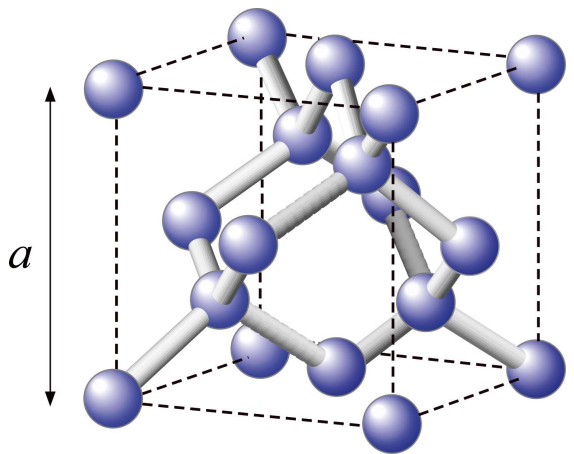

Fig. 3 Unit cell of the silicon cubic crystal. The lengths of edges are equal to the lattice parameter $a$. Apparently, 18 atoms are involved in the unit cell. However, the eight corner atoms are shared by eight adjacent unit cells. The six atoms on the plane are shared by two adjacent unit cells. One unit cell therefore contains eight $(=8 \times(1 / 8)$ $+6 \times(1 / 2)+4)$ atoms. ${ }^{13}$

$$
N=8 V / a^{3}
$$

The mass of the silicon sample $m$ is related to $N$ by

$$
m=N m(\mathrm{Si})
$$

where $m(\mathrm{Si})$ is the mass of a single $\mathrm{Si}$ atom. The relationship between the Avogadro constant $N_{\mathrm{A}}$ and $m(\mathrm{Si})$ is given by

$$
m(\mathrm{Si})=M / N_{\mathrm{A}},
$$

where $M$ is the molar mass of silicon. By combing Eqs. (4), (5) and (6), we obtain the Avogadro constant using

$$
N_{\mathrm{A}}=\frac{8 M V}{m a^{3}} .
$$

The XRCD method was therefore originally used to measure the Avogadro constant. The relationship of the Avogadro constant with the Planck constant is given by

$$
h=\frac{c M_{\mathrm{u}} A_{\mathrm{r}}(\mathrm{e}) \alpha^{2}}{2 R_{\infty}} \frac{1}{N_{\mathrm{A}}},
$$

where $c$ is the speed of light in vacuum, $M_{\mathrm{u}}$ is the molar mass constant, $A_{\mathrm{r}}(\mathrm{e})$ is the relative atomic mass of electron, $\alpha$ is the fine-structure constant, and $R$ is the Rydberg constant. ${ }^{14}$ The relative standard uncertainty of the term $c M_{\mathrm{u}} A_{\mathrm{r}}(\mathrm{e}) \alpha^{2} / R$ is estimated to be as small as $4.5 \times 10^{-10} .15$ When a value of $N_{\mathrm{A}}$ with, say, the relative uncertainty of $10^{-8}$ level is obtained, a value of $h$ with essentially the same uncertainty is therefore obtained.

In the following, details of measurements of the parameters to determine $h$ by the XRCD method are provided. As an example, a determination of $h$ by the National Metrology Institute of Japan (NMIJ) is also introduced. ${ }^{9}$

\section{1 ${ }^{28}$ Si-enriched crystal}

Natural silicon consists of three stable isotopes, ${ }^{28} \mathrm{Si},{ }^{29} \mathrm{Si}$, and ${ }^{30} \mathrm{Si}$, whose approximate isotopic abundances are $92 \%, 5 \%$, and $3 \%$, respectively. In Eqs. (6) and (7), the mean molar mass obtained from the measurements of the composition of each isotope in the crystal should be used. Several national metrology institutes measured the Planck constant $h$ using silicon crystals

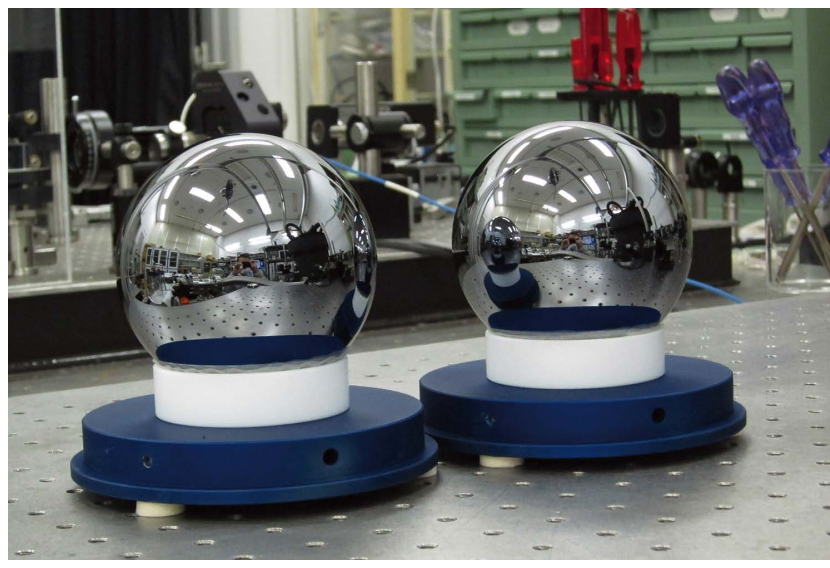

Fig. $41 \mathrm{~kg}{ }^{28} \mathrm{Si}$-enriched spheres manufactured from the AVO28 crystal. ${ }^{13}$ Left, AVO28-S5c; right, AVO28-S8c.

with natural isotopic abundance. However, the relative standard uncertainty of $h$ was on the order of $10^{-7}$, which was clearly larger than the possible stability of the mass of the international prototype of the kilogram over 100 years. The dominant uncertainty source was the molar mass determination. The smallest relative standard uncertainty achieved for the molar mass measurement of a silicon crystal with natural isotopic abundance was as large as $2.4 \times 10^{-7} .^{16}$ To solve this problem, a silicon crystal highly enriched in ${ }^{28} \mathrm{Si}$ was produced in the International Avogadro Coordination (IAC) project. ${ }^{13,17}$ The enrichment of the ${ }^{28} \mathrm{Si}$ isotope was higher than $99.99 \%$, and this crystal was referred to as AVO28. From the AVO28 crystal, samples were manufactured and used for determinations of the Planck constant.

\subsection{Sphere volume measurement}

To ensure the traceability to the SI, the volume measurement in the XRCD method should be traceable to the definition of the meter. Recent strategies for determining the volume from length measurement use a sphere because a sphere has the following advantages: (1) it is much less susceptible to be damaged than a cube or a cylinder, and (2) its volume is accurately determined on the basis of diameter measurement from many different directions when the deviation from a perfect spherical shape is small. $^{18}$ Furthermore, the mass of a sample should be comparable to that of the international prototype of the kilogram to measure the sample mass as accurately as possible. Therefore, $1 \mathrm{~kg}{ }^{28} \mathrm{Si}$-enriched spheres were used as samples for the XRCD method.

Figure 4 shows two $1 \mathrm{~kg}{ }^{28} \mathrm{Si}$-enriched spheres manufactured from the AVO28 crystal, named AVO28-S5c and AVO28-S8c. Figure 5 shows the locations of the two ${ }^{28} \mathrm{Si}$-enriched spheres in the AVO28 crystal. These two ${ }^{28} \mathrm{Si}$-enriched spheres were used by the IAC project to measure the Avogadro constant and subsequently to derive the Planck constant. Figure 6 shows a photograph of the optical interferometer used to measure the diameter of the ${ }^{28} \mathrm{Si}$-enriched spheres developed by Kuramoto et al at NMIJ. ${ }^{13,19}$ The principle of the diameter measurement is also shown in this figure. A sphere is placed in a fused-quartz Fabry-Perot etalon formed by two flat reference surfaces. The gaps between the reference surface and the adjacent surface of the sphere, $d_{1}$ and $d_{2}$, are measured by optical interferometry. The etalon distance $L$ is also measured by optical interferometry, and the sphere diameter $D$ is given by $D=L-\left(d_{1}+d_{2}\right)$. 


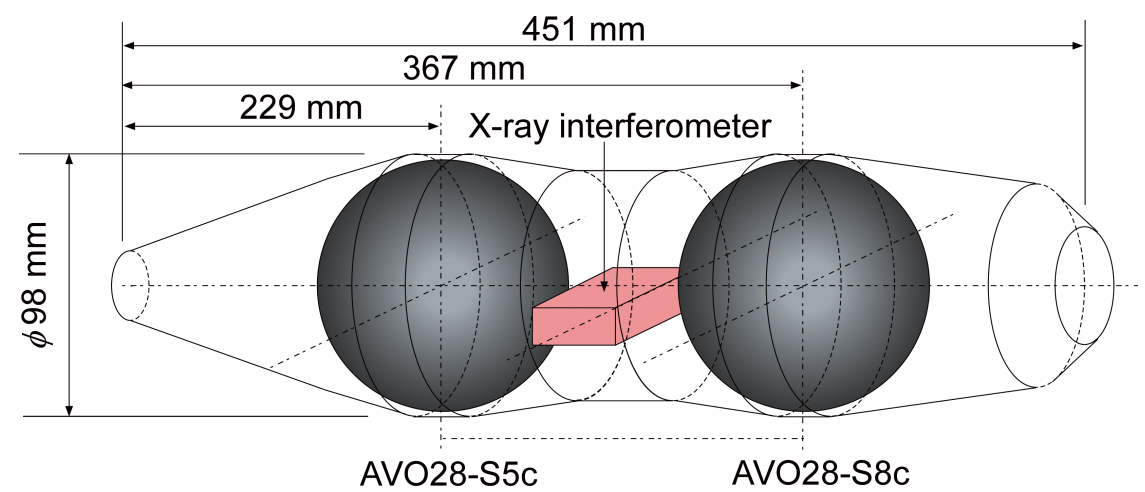

Fig. 5 Locations of the two ${ }^{28} \mathrm{Si}$-enriched spheres AVO28-S5c and AVO28-S8c and the x-ray interferometer in the AVO28 crystal.
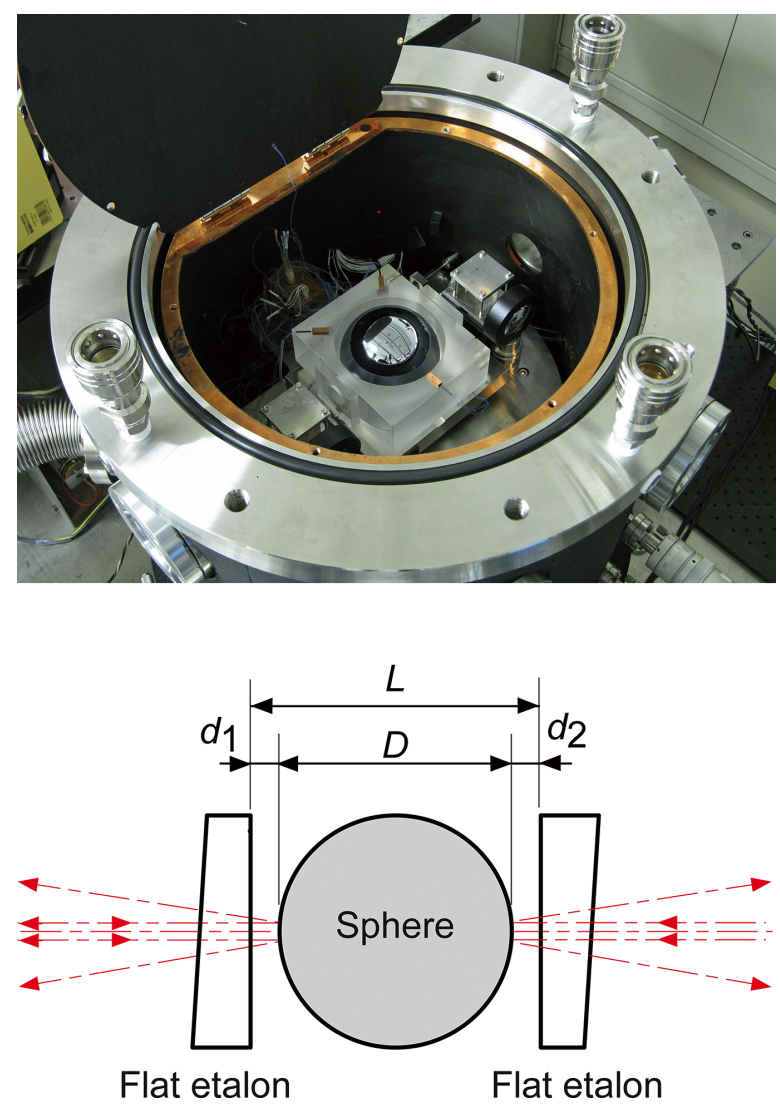

Fig. 6 Top: Optical interferometer to measure the diameter of the ${ }^{28} \mathrm{Si}$-enriched spheres developed by Kuramoto et al. ${ }^{13}$ Bottom: Principle of the diameter measurement using an etalon with flat reference surfaces.

Figure 7 explains the details of the interferometer. The sphere and etalon are installed in a vacuum chamber equipped with an active radiation shield to precisely control the sphere temperature. A laser beam from an external cavity diode laser is split into two beams (Beam 1 and Beam 2), which are then reflected by mirrors toward the opposite sides of the etalon. The light beams reflected from the inner surface of the etalon plate and the adjacent surface of the sphere interfere to produce concentric circular fringes. These are projected onto chargecoupled device cameras (CCD 1 and CCD 2). The fringes are analyzed by phase-shifting interferometry to measure $d_{1}$ and $d_{2}$, in which the phase shifts are produced by changing the optical frequency of the diode laser. ${ }^{20}$ The optical frequency is controlled on the basis of an optical frequency comb, which is used as the national length standard of Japan. ${ }^{21}$ To determine the etalon spacing $L$, the sphere is removed from the light path by a lifting device installed underneath the sphere, and a shutter intercepts Beam 1. Beam 2 passes through a hole in the lifting device, and the beams reflected from the two etalon plates produce fringes on the third CCD camera (CCD 3). These fringes are also analyzed by phase-shifting interferometry.

A sphere rotation mechanism installed under the sphere is used to measure the diameter from many different directions. In a set of diameter measurements, the diameter is measured from 145 directions distributed near-uniformly on the sphere surface. ${ }^{19}$ The set of diameter measurements is repeated, and between the sets, the sphere is rotated to distribute the starting point of each set of measurements to the vertices of a regular dodecahedron. Because the 10 directions defined by the vertices of a regular dodecahedron are uniformly distributed, the procedure therefore distributes all of the measurement points as uniformly as possible. The sphere volume is calculated from the mean diameter.

Figure 8 shows the three-dimensional plot of the distribution of the diameter measured from the 145 directions for AVO28S5c (left) and AVO28-S8c (right), where the deviation from the mean diameter is enhanced. The peak-to-valley values of the diameter were $69 \mathrm{~nm}$ for AVO28-S5c and $38 \mathrm{~nm}$ for AVO28S8c. The total number of measurement directions was 2320 for AVO28-S5c and 870 for AVO28-S8c. The relative standard uncertainty of the sphere volume measurement was estimated to be $2.0 \times 10^{-8}$ for AVO28-S5c and $2.2 \times 10^{-8}$ for AVO28-S 8 c. ${ }^{19}$

A different type of optical interferometer developed at the Physikalisch-Technische Bundesanstalt (PTB, Germany) was also used to measure the volume of AVO28-S5c and AVO28S8c. ${ }^{22}$ The comparison of the two interferometers is shown in Fig. 9. In the PTB interferometer, an etalon with spherical reference surfaces were used. The comparison of the mean diameters measured at PTB and NMIJ is also shown in Fig. 9. The diameters obtained using the two interferometers with different optical configurations show excellent agreement within their uncertainties.

As noted in Sect. 5·1, both AVO28-S5c and AVO28-S8c were used to measure the Avogadro constant and subsequently to derive the Planck constant. However, in the following, only results using AVO28-S5c are described as an example of the determination of $h$ by NMIJ to make the explanation of the results simple and straightforward. 


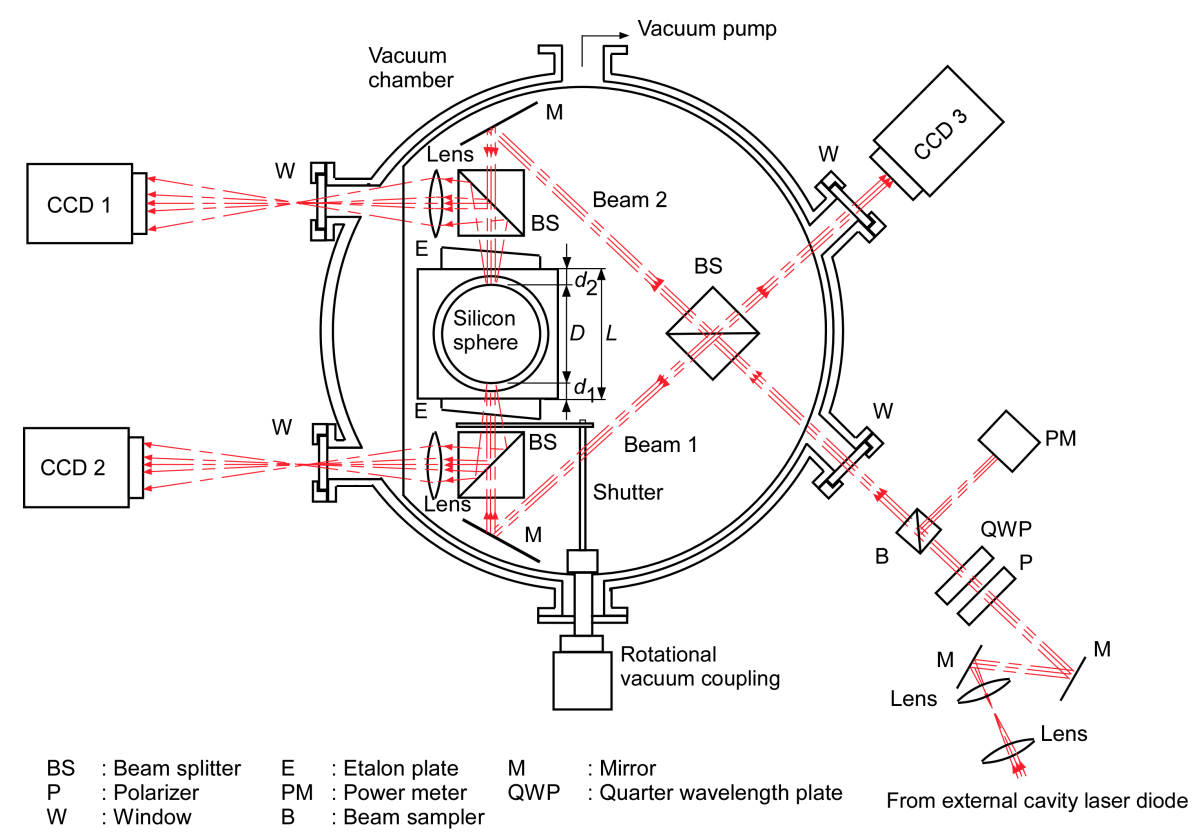

Fig. 7 Optical interferometer used to measure diameter of silicon spheres. ${ }^{19}$
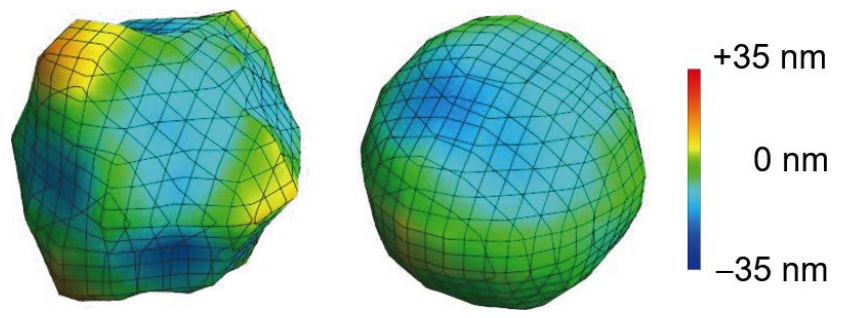

Fig. 8 Three-dimensional plots of the diameters of AVO28-S5c (left) and AVO28-S8c (right) measured using the optical interferometer by NMIJ. The peak-to-valley value of the diameter were $69 \mathrm{~nm}$ for $\mathrm{AVO} 28-\mathrm{S} 5 \mathrm{c}$ and $38 \mathrm{~nm}$ for AVO28-S8c. ${ }^{19}$

\subsection{Sphere mass measurement}

Because the masses of the ${ }^{28} \mathrm{Si}$-enriched spheres are almost $1 \mathrm{~kg}$, it is possible to determine the masses by directly comparing them with a $1 \mathrm{~kg}$ reference weight such as a $1 \mathrm{~kg}$ platinumiridium alloy weight or a 1-kg stainless steel weight. However, the volumes of the ${ }^{28} \mathrm{Si}$-enriched spheres are significantly larger than that of the reference weight owing to the differences in density $\rho$ among silicon $\left(\rho=2320 \mathrm{~kg} \mathrm{~m}^{-3}\right)$, platinum-iridium alloy $\left(\rho=21500 \mathrm{~kg} \mathrm{~m}^{-3}\right)$, and stainless steel $\left(\rho=8000 \mathrm{~kg} \mathrm{~m}^{-3}\right)$. When the masses are compared in air, the air density should therefore be measured for the air buoyancy correction. A strategy to accurately compare the masses is mass comparison in vacuum, in which the uncertainty contribution from the air buoyancy correction can be neglected.

At NMIJ, a vacuum mass comparator was used to determine the masses of the ${ }^{28} \mathrm{Si}$-enriched spheres. ${ }^{23}$ The mass of the platinum-iridium kilogram standards of NMIJ was used as the reference in the measurement. The mass of AVO28-S5c in vacuum was estimated with a standard uncertainty of $5.9 \mu \mathrm{g}$, corresponding to the relative standard uncertainty of $5.9 \times 10^{-9}$ for $1 \mathrm{~kg}$. Figure 10 shows the masses determined by BIPM, PTB and NMIJ. ${ }^{24}$ As shown in this figure, the masses determined by these institutes show excellent agreement within their uncertainties.
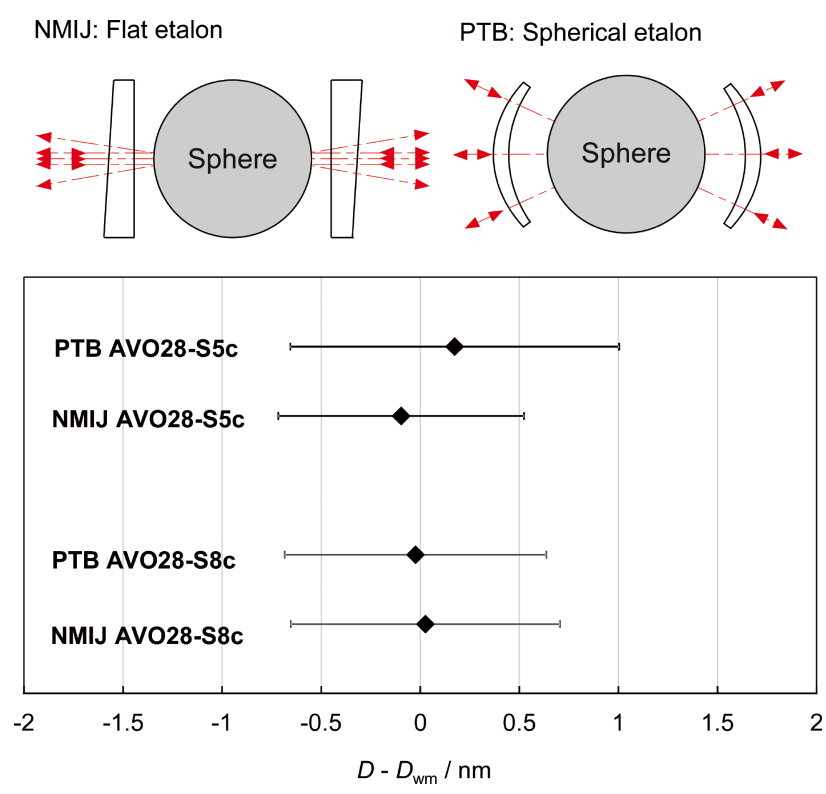

Fig. 9 Top: Optical configurations of the two interferometers of PTB and NMIJ. Bottom: Mean diameters of the ${ }^{28} \mathrm{Si}$-enriched spheres measured at PTB and NMIJ. The difference from the weighted mean diameter $D_{\text {wm }}$ calculated from the diameters measured at the two national metrology institutes is plotted. The horizontal error bars indicate the standard uncertainty of each value. ${ }^{19}$

\section{5•4 Surface characterization}

Equation (7) is established when the silicon sphere consists of only silicon crystal. However, the surface of the sphere is covered by a surface layer mainly consisting of $\mathrm{SiO}_{2}$. Figure 11 shows the surface layer model of ${ }^{28} \mathrm{Si}$-enriched spheres in vacuum derived on the basis of surface characterization by the IAC project. ${ }^{24}$ In addition to the $\mathrm{SiO}_{2}$ layer (OL), a chemisorbed water layer $(\mathrm{CWL})$ and a carbonaceous contamination layer (CL) are also present. The CWL is a chemically absorbed water 


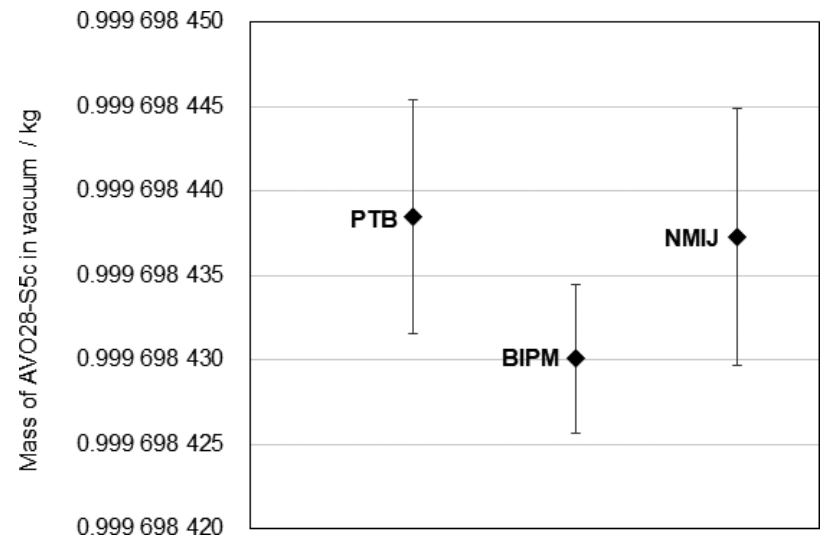

Fig. 10 Masses of AVO28-S5c in vacuum measured at BIPM, PTB and NMIJ. The vertical error bars indicate the standard uncertainty of each value. ${ }^{24}$

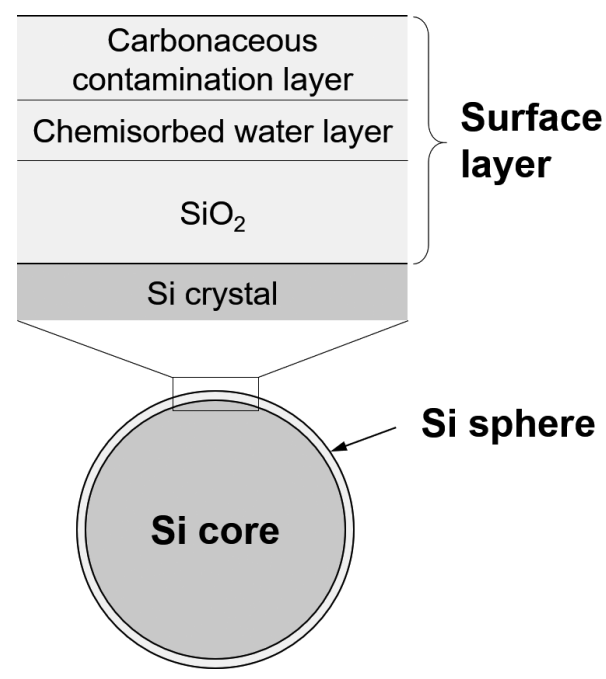

Fig. 11 Model of the surface layers of ${ }^{28} \mathrm{Si}$-enriched spheres in vacuum.

layer that remains under high vacuum conditions. The CL is a mixture of different hydrocarbons and is formed by different adsorbed gases and contaminants originating from the environment during the measurement, handling, storage and cleaning of the sphere. These layers are hereinafter collectively referred to as the surface layer (SL). Equation (7) should therefore be modified to

$$
N_{\mathrm{A}}=\frac{8 M V_{\text {core }}}{m_{\text {core }} a^{3}},
$$

where $m_{\text {core }}$ and $V_{\text {core }}$ are the mass and volume of the "Si core" part, which excludes the surface layer, respectively. To determine $V_{\text {core }}$ and $m_{\text {core }}$, the thickness and mass of the SL should be measured.

The surface layer was analyzed by various analysis methods such as x-ray reflectometry (XRR), X-ray fluorescence analysis (XRF), x-ray photoelectron spectroscopy (XPS), ellipsometry, and gravimetry to determine the thickness and mass of each layer. ${ }^{24}$ At NMIJ, an XPS system and an ellipsometer system were developed for the surface characterization. Details of the
Table 1 Mass, thickness, and density of each sublayer in vacuum of AVO28-S5c ${ }^{9}$

\begin{tabular}{lccr}
\hline Layer & Thickness/nm & Density/g cm & \multicolumn{1}{c}{ Mass $/ \mu \mathrm{g}$} \\
\hline OL & $1.24(9)$ & $2.2(1)$ & $75.2(6.3)$ \\
CL & $1.27(12)$ & $1.08(14)$ & $37.7(5.9)$ \\
CWL & $0.28(8)$ & $1.0(1)$ & $7.7(2.3)$ \\
SL & & & $120.6(8.9)$ \\
\hline
\end{tabular}

two systems are summarized by Zhang et al. ${ }^{25}$ and Fujita et al. ${ }^{26}$ For AVO28-S5c, the thicknesses of the CL and OL at 52 points on the sphere surface were measured using the XPS system. ${ }^{9}$ The 52 points were distributed near-uniformly on the sphere surface. The ellipsometer system was used to examine the uniformity of the thickness of the OL at 812 points on the sphere surface. ${ }^{9}$

Table 1 shows the results of the surface characterization of AVO28-S5c by NMIJ in 2017.9 The thickness of the CWL estimated from the coefficient of water adsorption to $\mathrm{Si}$ surface reported by Mizushima ${ }^{27}$ is also listed in this table. The total thickness of the surface layer was about $3 \mathrm{~nm}$.

\subsection{Volume and mass of $\mathrm{Si}$ core}

The diameter measured using the optical interferometer is slightly different from the core diameter owing to the phase retardation of the laser beam by the surface layer. ${ }^{20}$ To derive the core diameter from the measured diameter, the total phase retardation on reflection at the sphere surface is calculated from the thickness and optical constants of each sublayer. The core volume $V_{\text {core }}$ is calculated from the mean core diameter $D_{\text {core }}$ as $V_{\text {core }}=(\pi / 6) D_{\text {core }}{ }^{3}$.

The diameter measured by the NMIJ optical interferometer described in Sect. 5.2 was therefore corrected to the core diameter. For the correction, the phase retardation was determined from the results of the surface characterization by NMIJ described in Sect. 5.4. The relative standard uncertainty of the $V_{\text {core }}$ determination of AVO28-S5c was estimated to be $2.0 \times 10^{-8} .9$

On the other hand, the core mass $m_{\text {core }}$ is determined from the mass of the sphere including the surface layer measured using a mass comparator $m_{\text {sphere }}$ and the mass of the surface layer evaluated by the surface characterization $m_{\mathrm{SL}}$ by

$$
m_{\text {core }}=m_{\text {sphere }}-m_{\mathrm{SL}}+m_{\text {deficit }}
$$

where $m_{\text {deficit }}$ is the effect of point defects and impurities on the core mass. The value of $m_{\text {deficit }}$ of AVO28-S5c was estimated to be 3.6(3.6) $\mu \mathrm{g} .{ }^{24}$

The mass of the surface layer under vacuum $m_{\mathrm{SL}}$ is given by

$$
m_{\mathrm{SL}}=m_{\mathrm{OL}}+m_{\mathrm{CL}}+m_{\mathrm{CWL}}
$$

where $m_{\mathrm{OL}}, m_{\mathrm{CL}}$, and $m_{\mathrm{CWL}}$ are the masses of the OL, CL, and CWL, respectively. The masses are calculated from the thickness and density of each sublayer.

The mass of each sublayer of AVO28-S5c determined by NMIJ is summarized in Table 1 along with the density and thickness of each layer. The total mass of the surface layer was estimated to be $120.6(8.9) \mu \mathrm{g} .{ }^{9}$ This uncertainty corresponds to the relative standard uncertainty of $8.9 \times 10^{-9}$ for $1 \mathrm{~kg}$.

\subsection{Lattice constant measurement}

The lattice constant measurement of silicon crystals can be 


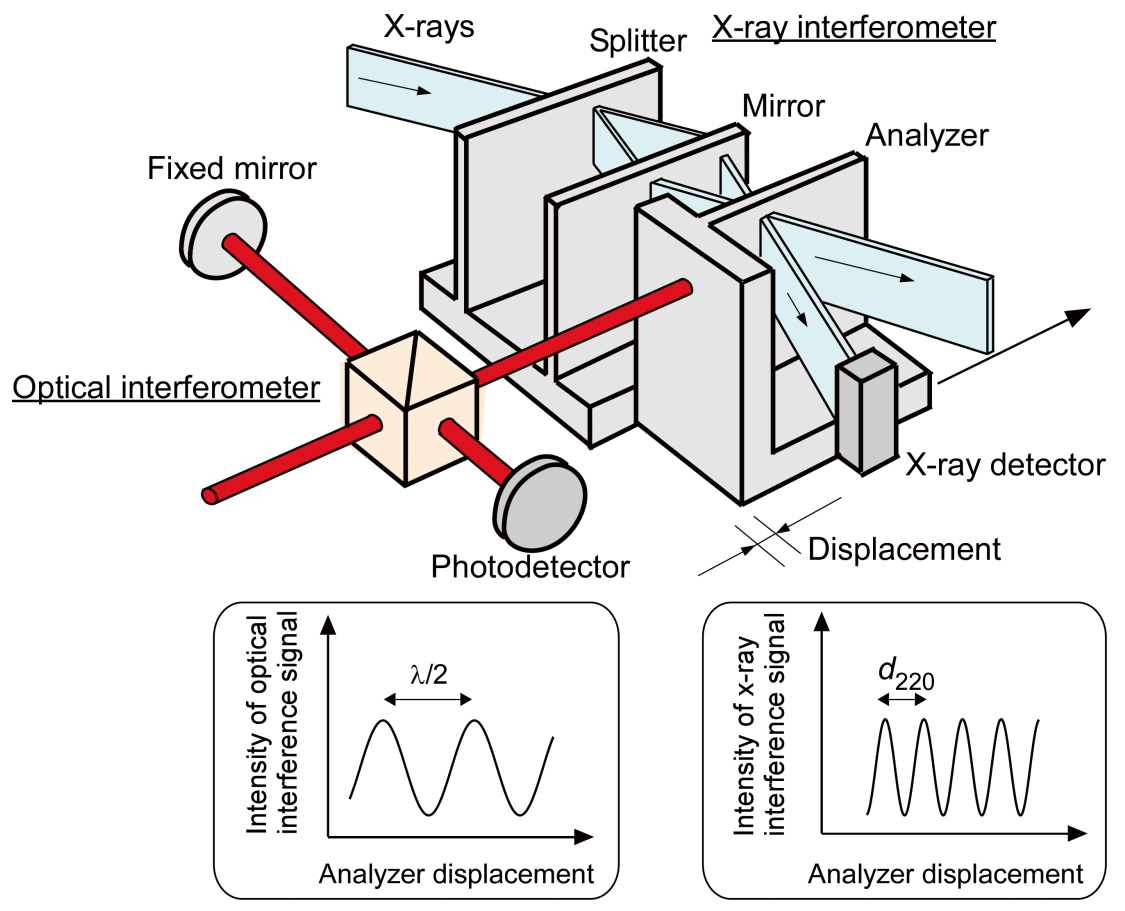

Fig. 12 Principle of the x-ray interferometer to measure the lattice constant. While the analyzer crystal is moved, its displacement is measured using the optical interferometer by counting the optical fringes having a period of $\lambda / 2$. Simultaneously, the x-ray fringes having a period of $d_{220}$ are also counted. $d_{220}$ is determined on the basis of the laser wavelength $\lambda$ by comparing the counts of $\mathrm{x}$-ray and optical interference fringes.

measured accurately using the combination of x-ray and optical interferometers. Figure 12 shows the measurement principle. The X-ray interferometer consists of three Si crystal blades that are cut so that the $\{220\}$ planes are orthogonal to the blade faces. An x-ray beam is split into two beams by the first blade (splitter). The two beams are split again by the second blade (mirror) and recombined by the third blade (analyzer). The $\mathrm{x}$-ray interference fringes produced by the beam recombination are integrated in an x-ray detector. When the analyzer is displaced with respect to the other two blades in a direction orthogonal to the $\{220\}$ planes, a periodic intensity variation of the $\mathrm{x}$-ray interference signal is observed, as shown in Fig. 12. The period of the variation is the lattice spacing of $\{220\}, d_{220}$, and the displacement of the analyzer is measured using the optical interferometer. The fundamental concept of the combination of the $\mathrm{x}$-ray and optical interferometers is to measure the unknown $d_{220}$ on the basis of the known wavelength of the laser used in the optical interferometer $\lambda$. The displacement of the analyzer is measured by counting the number of the optical interference fringes having a period of $\lambda / 2$. Simultaneously, the number of the $x$-ray fringes having a period of $d_{220}$ is also counted. The lattice spacing $d_{220}$ is determined by comparing the counts of the x-ray and optical interference fringes. The lattice constant $a$ is determined as $a=\sqrt{8} d_{220}$, where $\sqrt{8}$ accounts for the different spacings of the $\{100\}$ and $\{220\}$ planes. Details of the lattice constant measurement are summarized by Ferroglio et al. ${ }^{28}$

An x-ray interferometer was manufactured from the AVO28 crystal, as shown in Fig. 5. The lattice constant of the x-ray interferometer was measured at the Istituto Nazionale di Ricerca Metrologica (INRiM, Italy) by Massa et al..$^{29}$ Since contaminants such as carbon and nitrogen strain the crystal lattice, a contamination gradient in the crystal makes the lattice parameters of the sphere different from that of the x-ray interferometer. To measure the contamination gradient, samples were taken from the AVO28 crystal around the sphere. The impurity concentrations in the samples were measured by infrared spectroscopy. ${ }^{30}$ The lattice constant of AVO28-S5c was then calculated with a relative standard uncertainty of $1.8 \times 10^{-9}$ by taking into account the different contaminations of the sphere and the $\mathrm{x}$-ray interferometer. ${ }^{24}$

\subsection{Molar mass measurement}

The molar mass of a silicon crystal material is given by

$$
M=\sum_{\mathrm{n}=28}^{30} M\left({ }^{\mathrm{n}} \mathrm{Si}\right) x_{\mathrm{n}}
$$

where $M\left({ }^{\mathrm{n}} \mathrm{Si}\right)$ is the molar mass of the silicon isotope ${ }^{\mathrm{n}} \mathrm{Si}$ and $x_{\mathrm{n}}$ is the amount-of-substance fraction of ${ }^{n} \mathrm{Si}$. The Institute of Reference Materials and Measurements (IRMM) measured $x_{\mathrm{n}}$ of the ${ }^{28} \mathrm{Si}$-enriched crystal AVO28 by gas mass spectrometry, where samples prepared from the AVO28 crystal were converted to $\mathrm{SiF}_{4}$ gas by several chemical processes and the ratios of the ion currents from ${ }^{28} \mathrm{SiF}_{3}{ }^{+},{ }^{29} \mathrm{SiF}_{3}{ }^{+}$, and ${ }^{30} \mathrm{SiF}_{3}{ }^{+}$were measured. ${ }^{31}$ However, a small amount of natural silicon was estimated to be contaminated during the conversion processes, and the effect of the contamination on the ${ }^{28} \mathrm{Si}$ fraction measurement could not be accurately corrected. Furthermore, owing to the extremely high isotopic enrichment, the range in which isotope ratios had to be determined was extremely wide: $1 \times 10^{-6} \leq x\left({ }^{\mathrm{n}} \mathrm{Si}\right) / x\left({ }^{28} \mathrm{Si}\right) \leq 1$. This caused many difficulties owing to several issues such as detector linearity and dynamic range, limiting improvements regarding the measurement uncertainty.

To overcome these difficulties, a new strategy based on isotope dilution mass spectrometry combined with multicollector inductively coupled plasma mass spectrometry has been 
developed at PTB. ${ }^{32}$ This strategy does not directly measure $x\left({ }^{28} \mathrm{Si}\right)$. Instead, $x\left({ }^{29} \mathrm{Si}\right)$ and $x\left({ }^{30} \mathrm{Si}\right)$ are measured using an enriched spike of ${ }^{30} \mathrm{Si}$. Then, $x\left({ }^{28} \mathrm{Si}\right)$ is obtained indirectly as $x\left({ }^{28} \mathrm{Si}\right)=1-x\left({ }^{29} \mathrm{Si}\right)-x\left({ }^{30} \mathrm{Si}\right)$. This significantly narrows the isotope ratio measurement range. In addition, the conversion of the ${ }^{28} \mathrm{Si}$-enriched crystal into a liquid sample introduced to the mass spectrometer is carried out quantitatively in a single step by using alkaline solutions, avoiding cumulative contaminations by natural silicon. With these improvements, it has become possible to markedly improve the accuracy of the molar mass measurement.

To determine the molar mass of AVO28-S5c, small samples were taken from the AVO28 crystal from both sides of the sphere. The molar masses of the samples were measured by PTB, NMIJ, and the National Institute of Standards and Technology (NIST, USA), from which the molar mass of AVO28-S5c was estimated with a relative standard uncertainty of $5 \times 10^{-9} \cdot .^{24}$

\subsection{Avogadro constant and Planck constant}

NMIJ determined the Avogadro constant $N_{\mathrm{A}}$ in 2017 from their measurements of $V_{\text {core }}$ and $m_{\mathrm{SL}}$ of AVO28-S5c by combining $a, m_{\text {deficit }}$, and $M$ measured by the IAC project. ${ }^{9}$ This $N_{\mathrm{A}}$ was converted to the Planck constant $h$ using Eq. (8). The relative standard uncertainty of the $h$ determination was $2.4 \times 10^{-8}$. Table 2 shows the uncertainty budget of the determination of $h$. The largest uncertainty source was the determination of $V_{\text {core }}$.

\section{Determination of the Planck Constant in the New Definition of the Kilogram}

On the basis of the progress in the accuracy and consistency of the determinations of the Planck constant by the XRCD method

Table 2 Uncertainty budget for the determination of the Planck constant $h$ using AVO28-S5c by NMIJ in $2017^{9}$

\begin{tabular}{lc}
\hline \multicolumn{1}{c}{ Quantity } & $\begin{array}{c}\text { Relative standard } \\
\text { uncertainty in } h\end{array}$ \\
\hline Core volume, $V_{\text {core }}$ & $2.0 \times 10^{-8}$ \\
Mass of the surface layer, $m_{\mathrm{SL}}$ & $8.9 \times 10^{-9}$ \\
Relative atomic mass of silicon, $A_{\mathrm{r}}(\mathrm{Si})$ & $5.4 \times 10^{-9}$ \\
Lattice parameter, $a$ & $5.5 \times 10^{-9}$ \\
Mass deficit due to impurities and vacancies, $m_{\text {deficit }}$ & $3.8 \times 10^{-9}$ \\
Planck constant, $h$ & $2.4 \times 10^{-8}$ \\
\hline
\end{tabular}

and the Kibble balance technique, the Comité International des Poids et Mesures (CIPM) decided to undertake all the necessary steps to proceed with the adoption of the new definition of the kilogram based on a fixed value of the Planck constant $h$ in 2017. ${ }^{10}$

The Task Group on Fundamental Constants of the Committee on Data for Science and Technology (CODATA TGFC) periodically provides a self-consistent set of internationally recommended values of the basic constants and conversion factors of physics and chemistry to the scientific and technological communities. In 2017, the CODATA TGFC provided an adjusted value of $h$ used in the new definition of the kilogram. ${ }^{33,34}$ The eight data used in the adjustment are listed in Table 3. Figure 13 shows the eight data and the adjusted $h$ value (CODATA 2017). NMIJ-17 is the $h$ value measured by NMIJ in $2017,{ }^{9}$ the details of which are introduced in this paper. NIST-15, NIST-17, NRC-17, and LNE-17 were measured by NIST, the National Research Council (NRC, Canada) and the Laboratoire National de Métrologie et d'Essais (LNE, France), respectively, using the Kibble balances. ${ }^{35-38}$ IAC-11, IAC-15, and IAC-17 were measured by the International Avogadro Coordination project by the XRCD method. ${ }^{24,39}$ The $h$ value obtained by the CODATA adjustment is $h=6.626070150(69)$ $\times 10^{-34} \mathrm{~J} \mathrm{~s}$, with a relative standard uncertainty of $1.0 \times 10^{-8} .33,34$

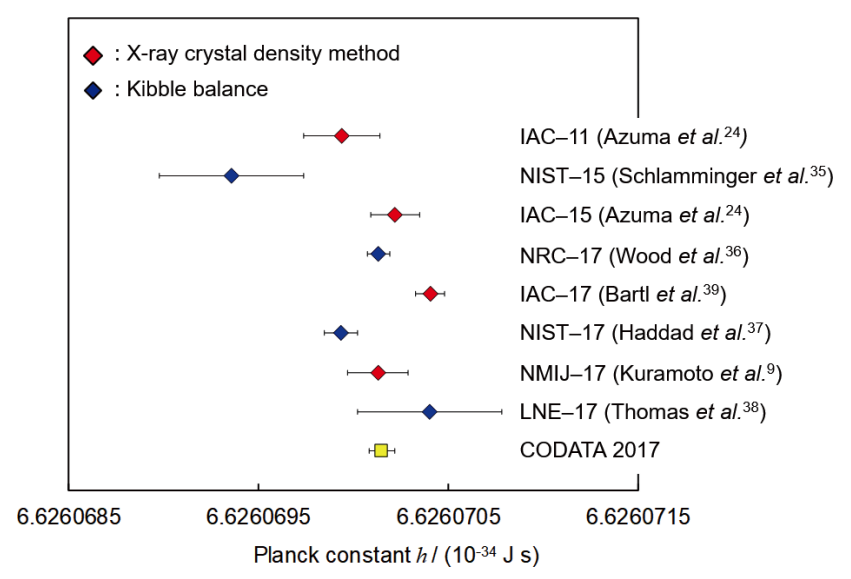

Fig. 13 Eight key data for the determination of the Planck constant $h$ in the CODATA 2017 Special Adjustment of the Fundamental Constants (CODATA2017, $\square$ ). .33,34 $^{33}$ The numerical value of $h$ in the CODATA2017 without uncertainty was accepted to establish the new definition of the kilogram in 2018. ${ }^{10}$ A complete list of input data in the CODATA 2017 Special Adjustment is provided by Mohr et al. ${ }^{34}$

Table 3 Details of the eight data used to determine the Planck constant $h$ for the new definition of the kilogram ${ }^{33}$ (See Mohr et al..$^{34}$ for a complete list of input data)

\begin{tabular}{|c|c|c|c|c|}
\hline Source (Year) & Data ID & Research institute & Method & $\begin{array}{l}\text { Relative standard } \\
\text { uncertainty in } h\end{array}$ \\
\hline Schlamminger et al. $(2015)^{35}$ & NIST-15 & National Institute of Standards and Technology (USA) & Kibble balance & $5.7 \times 10^{-8}$ \\
\hline Wood et al. $(2017)^{36}$ & NRC-17 & National Research Council (Canada) & Kibble balance & $9.1 \times 10^{-9}$ \\
\hline Haddad et al. $(2017)^{37}$ & NIST-17 & National Institute of Standards and Technology (USA) & Kibble balance & $1.3 \times 10^{-8}$ \\
\hline Thomas et al. $(2017)^{38}$ & LNE-17 & Laboratoire national de métrologie et d'essais (France) & Kibble balance & $5.7 \times 10^{-8}$ \\
\hline Azuma et al. $(2015)^{24}$ & IAC-11 & International Avogadro Coordination project & XRCD method & $3.0 \times 10^{-8}$ \\
\hline Azuma et al. $(2015)^{24}$ & IAC-15 & International Avogadro Coordination project & XRCD method & $2.0 \times 10^{-8}$ \\
\hline Bartl et al. $(2017)^{39}$ & IAC-17 & International Avogadro Coordination project & XRCD method & $1.2 \times 10^{-8}$ \\
\hline Kuramoto et al. $(2017)^{9}$ & NMIJ-17 & National Metrology Institute of Japan (Japan) & XRCD method & $2.4 \times 10^{-8}$ \\
\hline
\end{tabular}

a. Data name given in the CODATA 2017 Special Adjustment of the Fundamental Constants. ${ }^{33}$ 
The data set shown in Table 3 and Fig. 13 fulfills the requirements by the CCM noted in Sect. 3. In response to this, at the Conférence Générale des Poids et Mesures (CGPM) held in 2018, the following new definition of the kilogram was adopted. ${ }^{10}$ This new definition came into effect from May 20, 2019.

The kilogram, symbol $\mathrm{kg}$, is the SI unit of mass. It is defined by taking the fixed numerical value of the Planck constant $h$ to be $6.62607015 \times 10^{-34}$ when expressed in the unit $\mathrm{J} \mathrm{s}$, which is equal to $\mathrm{kg} \mathrm{m}^{2} \mathrm{~s}^{-1}$, where the meter and the second are defined in terms of the speed of light in vacuum and the unperturbed ground-state hyperfine transition frequency of ${ }^{133} \mathrm{Cs}$ atom.

\section{New Definition of the Mole Based on the Avogadro Constant}

In conjunction with the revision of the definition of the kilogram, the definition of the mole was also revised to the following definition based on the Avogadro constant. ${ }^{10}$

The mole, symbol mol, is the SI unit of amount of substance. One mole contains exactly $6.02214076 \times 10^{23}$ elementary entities. This number is the fixed numerical value of the Avogadro constant, $N_{\mathrm{A}}$, when expressed in the unit $\mathrm{mol}^{-1}$ and is called the Avogadro number.

The definition of the mole is presently independent of the definition of the kilogram and the mass of a specific nuclide such as ${ }^{12} \mathrm{C}$. It only depends on the number of elementary particles, being straightforward and easy to understand. The $N_{\mathrm{A}}$ value used in this new definition was determined in the CODATA 2017 adjustment, where the eight data in Table 3 were used to determine both $N_{\mathrm{A}}$ and $h$ using Eq. (8). ${ }^{33,34}$ The eight data therefore played an essential role in deriving the new definitions of both the kilogram and the mole.

\section{Impact of the Redefinition of the Kilogram}

8-1 Dissemination of the kilogram under the new definition

Under the previous definition of the kilogram, national prototypes of the kilogram of each country were sent to BIPM in a cycle of about 30 to 40 years, and their masses were measured on the basis of the international prototype of the kilogram. Each national metrology institute measured the mass of various weights on the basis of the national prototype of the kilogram and supplied these weights as mass standards in each country.

Under the new definition, it is in principle possible for each national metrology institute to realize the kilogram independently. To ensure the consistency among the realizations of the kilogram by national metrology institutes, an international comparison of the realizations is presently being conducted. This comparison is an essential step to the dissemination of the kilogram based on the individual realization by each national metrology institute. ${ }^{40}$

After the confirmation of the international consistency, the XRCD method and the Kibble balance will be used for the individual realizations. ${ }^{41}$ NMIJ will realize the kilogram by the XRCD method using the ${ }^{28}$ Si-enriched spheres. By combining Eqs. (8), (9), and (10), we obtain

$$
m_{\text {sphere }}=\frac{2 h R_{\infty}}{c \alpha^{2}} \frac{A_{\mathrm{r}}(\mathrm{Si})}{A_{\mathrm{r}}(\mathrm{e})} \frac{8 V_{\text {core }}}{a^{3}}+m_{\mathrm{SL}}-m_{\text {deficit }}
$$

The relative uncertainty of $R_{\infty} /\left(\alpha^{2} A_{\mathrm{r}}(\mathrm{e})\right)$ is estimated to be as

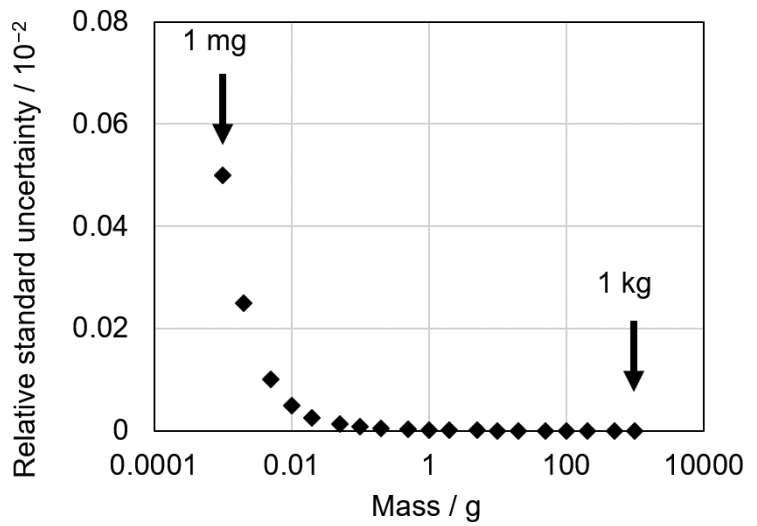

Fig. 14 Relative standard uncertainties of the masses of standard weights estimated from the maximum possible error in OIML R-111. ${ }^{45}$ Obtaining a standard weight with a mass less than $1 \mathrm{~kg}$ usually requires submultiples of a primary $1 \mathrm{~kg}$ standard. Each time a mass is subdivided, the relative uncertainties of the masses of the weights calibrated by the subdivision process accrues progressively.

small as of $4.0 \times 10^{-10},{ }^{42}$ and the value $c$ is exactly defined in the new SI. ${ }^{1}$ The mass of the sphere can therefore be determined from the measurements of $V_{\text {core }}, m_{\mathrm{SL}}, A_{\mathrm{r}}(\mathrm{Si}), a$, and $m_{\text {deficit }}$ on the basis of the Planck constant $h$ in the new definition of the kilogram. ${ }^{43}$ The ${ }^{28} \mathrm{Si}$-enriched spheres will therefore be used as artifacts to realize the kilogram. On the basis of the masses of the ${ }^{28} \mathrm{Si}$-enriched spheres, the masses of $1 \mathrm{~kg}$ platinum-iridium weights and stainless steel weights are determined. These weights will be used to measure the mass of standard weights disseminated to various scientific and industrial fields in Japan.

8.2 Small mass measurement based on the Planck constant Under the previous definition of the kilogram, standard weights calibrated on the basis of the international prototype of the kilogram were essential to ensure the metrological traceability of mass measurements. Obtaining a standard weight with a mass of less than $1 \mathrm{~kg}$ usually requires submultiples of a primary $1 \mathrm{~kg}$ standard. ${ }^{44}$ Each time a mass is subdivided, the relative uncertainty of the mass of the weight calibrated by the subdivision process accrues progressively. Figure 14 shows the relative standard uncertainties of the masses of weights with various masses estimated from maximum possible error used in legal metrology. ${ }^{45}$ At the level of $1 \mathrm{~kg}$, the relative standard uncertainty of the mass is $8 \times 10^{-6}$. However, by the time the mass is subdivided to $1 \mathrm{mg}$, the relative standard uncertainty is approximately 2400000 times.

On the other hand, the new definition enables us to realize the kilogram using any measurement technology that relates the Planck constant to the kilogram. ${ }^{1}$ Mass in any range can therefore be directly measured on the basis of the definition of the kilogram without using the standard weights. An example of such measurement techniques is an electrostatic force balance for mass measurements in the $\mathrm{mg}$ range. ${ }^{46}$ In the electrostatic force balance, electrostatic force is generated and is balanced with the gravity force acting on a sample, where the electrostatic force is measured on the basis of the Planck constant. The electrostatic force balance can therefore measure the mass of the sample on the basis of the new definition of the kilogram without using the standard weights. Recently, Shaw et al. have extended the measurement range by this technique to the $\mu \mathrm{g}$ range. ${ }^{47}$ 


\section{Impact of the Redefinition of the Mole}

The mass of an atom is extremely small. When we represent the mass of an atom, the relative atomic mass defined as the ratio of the mass of the atom to the unified atomic mass unit is therefore used. The unified atomic mass unit is defined as $1 / 12$ of the mass of a ${ }^{12} \mathrm{C}$ atom. The relative atomic mass of various nuclides is an essential intellectual infrastructure used in many scientific fields. The redefinition of the mole does not affect the value of the relative atomic mass. For example, the relative atomic mass of ${ }^{12} \mathrm{C}, A_{\mathrm{r}}\left({ }^{12} \mathrm{C}\right)$, is exactly 12 before and after the redefinition. However, the value of the molar mass constant $M_{\mathrm{u}}$ used to derive the molar mass from the relative atomic mass was slightly changed by the redefinition. Under the previous definition of the mole, $M_{\mathrm{u}}$ was defined as $1 \times 10^{-3} \mathrm{~kg} \mathrm{~mol}^{-1}$, and the molar mass of ${ }^{12} \mathrm{C}$ was exactly $12 \times 10^{-3} \mathrm{~kg} \mathrm{~mol}^{-1}$. On the other hand, under the present definitions of the kilogram and the mole, $M_{\mathrm{u}}$ is given by

$$
M_{\mathrm{u}}=\frac{2 N_{\mathrm{A}} h}{c} \frac{R_{\infty}}{\alpha^{2} A_{\mathrm{r}}(\mathrm{e})} .
$$

Because the fine-structure constant $\alpha$, the Rydberg constant $R_{\infty}$, and the relative atomic mass of electron $A_{\mathrm{r}}(\mathrm{e})$ are measured quantities, $M_{\mathrm{u}}$ changes depending on these values. From these values in the CODATA 2018 adjustment, $M_{\mathrm{u}}$ is estimated to be $0.99999999965(30) \times 10^{-3} \mathrm{~kg} \mathrm{~mol}^{-1}{ }^{42}$ The molar mass of ${ }^{12} \mathrm{C}$ is presently therefore not exactly $12 \times 10^{-3} \mathrm{~kg}$ but 11.999999 $9958(36) \times 10^{-3} \mathrm{~kg}$ with a relative standard uncertainty of $3.0 \times$ $10^{-10}$.

The relative atomic masses of some elements have been determined with relative uncertainties of the $10^{-10}$ level. ${ }^{48}$ The changes in the molar masses of such elements due to the redefinition is therefore not negligible. However, the changes are very small and can be ignored in most chemical measurements and the realization of the mole by national metrology institutes. ${ }^{49}$

\section{Impact of the Redefinition on New Science and Technology}

Looking back on the history of modern science, the role of the SI is not a simple common measurement scale but a common intellectual infrastructure of human beings, which generates technological innovation. For example, the definition of the meter based on the speed of light in vacuum enables us to perform accurate length measurements on the order of nanometers, building the foundation for nanotechnology. In general measurements, the effect of the redefinition of the kilogram and the mole is almost negligible at the moment. However, under the new definitions, in principle, it is possible to measure mass and amount of substance at the atomic level. It may not be so far in the future that new science and technology develops with the redefinition acting as a trigger to bring about dramatic progress in industrial technology and daily life.

\section{Acknowledgements}

The research work to redefine the kilogram and the mole at NMIJ was partly supported by the Japan Society for the Promotion of Science (JSPS) KAKENHI Grant Numbers 24360037, 16H03901, and 20H02630.

\section{References}

1. "The International System of Units (SI)", 9th ed., 2019, Bureau International des Poids et Mesures, https://www. bipm.org/utils/common/pdf/si-brochure/SI-Brochure-9-EN. pdf.

2. R. Davis, P. Barat, and M. Stock, Metrologia, 2016, 53, A12.

3. T. Quinn, Studies in History and Philosophy of Science, 2017, 65-66, 8 .

4. M. Milton, Metrologia, 2013, 50, 158.

5. G. Girard, Metrologia, 1994, 31, 317.

6. R. Davis, Metrologia, 2003, 40, 299.

7. M. Milton, R. Davis, and N. Fletcher, Metrologia, 2014, 51, R21.

8. I. A. Robinson and S. Schlamminger, Metrologia, 2016, 53, A46.

9. N. Kuramoto, S. Mizushima, L. Zhang, K. Fujita, Y. Azuma, A. Kurokawa, S. Okubo, H. Inaba, and K. Fujii, Metrologia, 2017, 54, 716.

10. M. Stock, R. Davis, E. de Mirandés, and M. J. Milton, Metrologia, 2019, 56, 022001.

11. L. S. Chao, S. Schlamminger, D. B. Newell, J. R. Pratt, F. Seifert, X. Zhang, G. Sineriz, M. Liu, and D. Haddad, Am. J. Phys., 2015, 83, 913.

12. A. Eichenberger H. Baumann, B. Jeanneret, B. Jeckelmann, P. Richard, and W. Beer, Metrologia, 2011, 48, 133.

13. Mass Standards Group of the National Metrology Institute of Japan/National Institute of Advanced Industrial Science and Technology, https://unit.aist.go.jp/riem/mass-std/.

14. I. M. Mills, P. J. Mohr, T. J. Quinn, B. N. Taylor, and E. R. Williams, Metrologia, 2005, 42, 71.

15. P. J. Mohr, D. B. Newell, and B. N. Taylor, J. Phys. Chem. Ref. Data, 2016, 45, 043102.

16. S. Valkiers, G. Mana, K. Fujii, and P. Becker, Metrologia, 2011, 48, S26.

17. B. Andreas, Y. Azuma, G. Bartl, P. Becker, H. Bettin, M. Borys, I. Busch, P. Fuchs, K. Fujii, H. Fujimoto, E. Kessler, M. Krumrey, U. Kuetgens, N. Kuramoto, G. Mana, E. Massa, S. Mizushima, A. Nicolaus, A. Picard, A. Pramann, O. Rienitz, D. Schiel, S. Valkiers, A. Waseda, and S. Zakel, Metrologia, 2011, 48, S1.

18. D. P. Johnson, J. Res. Natl. Bur. Stand., 1974, 78A, 41.

19. N. Kuramoto, Y. Azuma, H. Inaba, and K. Fujii, Metrologia, 2017, 54, 193.

20. N. Kuramoto, K. Fujii, and K. Yamazawa, Metrologia, 2011, 48, S83.

21. H. Inaba, Y. Nakajima, F. L. Hong, K. Minoshima, J. Ishikawa, A. Onae, H. Matsumoto, M. Wouters, B. Warrington, and N. Brown, IEEE Trans. Instrum. Meas., 2009, 58, 1234.

22. G. Bartl, H. Bettin, M. Krystek, T. Mai, A. Nicolaus, and A. Peter, Metrologia, 2011, 48, S96.

23. S. Mizushima, N. Kuramoto, L. Zhang, and K. Fujii, IEEE Trans. Instrum. Meas., 2017, 66, 1275.

24. Y. Azuma, P. Barat, G. Bartl, H. Bettin, M. Borys, I. Busch, L. Cibik, G. D’Agostino, K. Fujii, H. Fujimoto, A. Hioki, M. Krumrey, U. Kuetgens, N. Kuramoto, G. Mana, E. Massa, R. Meeß, S. Mizushima, T. Narukawa, A. Nicolaus, A. Pramann, S. A. Rabb, O. Rienitz, C. Sasso, M. Stock, R. D. Vocke Jr., A. Waseda, S. Wundrack, and S. Zakel, Metrologia, 2015, 52, 360.

25. L. Zhang, N. Kuramoto, Y. Azuma, A. Kurokawa, and K. Fujii, IEEE Trans. Instrum. Meas., 2017, 66, 1297. 
26. K. Fujita, N. Kuramoto, Y. Azuma, S. Mizushima, and K. Fujii, IEEE Trans. Instrum. Meas., 2017, 66, 1283.

27. S. Mizushima, Metrologia, 2004, 41, 137.

28. L. Ferroglio, G. Mana, and E. Massa, Opt. Express, 2008, $16,16877$.

29. E. Massa, C. P. Sasso, G. Mana, and C. Palmisaon, J. Phys. Chem. Ref. Data, 2015, 44, 031208.

30. S. Zakel, Metrologia, 2011, 48, S14.

31. E. Bulska, M. N. Drozdov, G. Mana, A. Pramann, O. Rienitz, P. Sennkov, and S. Valkiers, Metrologia, 2011, 48, S32.

32. O. Rienitz, A. Pramann, and D. Schiel, Int. J. Mass Spectrom., 2010, 289, 47.

33. D. B. Newell, F. Cabiati, J. Fischer, K. Fujii, S. G. Karshenboim, H. S. Margolis, E. de Mirandés, P. J. Mohr, F. Nez, K. Pachucki, T. J. Quinn, B. N. Taylor, M. Wang, B. M. Wood, and Z. Zhang, Metrologia, 2018, 55, L13.

34. P. J. Mohr, D. B. Newell, B. N. Taylor, and E. Tiesinga, Metrologia, 2018, 55, 125.

35. S. Schlamminger, R. L. Stiner, D. Haddad, D. B. Newell, F. Seifert, L. S. Chao, R. Liu, E. R. Williams, and J. R. Pratt, Metrologia, 2015, 52, L5.

36. B. Wood, C. A. Sanchez, R. G. Green, and J. O. Liard, Metrologia, 2017, 54, 399.

37. D. Hadded, F. Seifert, L. S. Chao, A. Possolo, D. B. Newell, J. R. Pratt, C. J. Williams, and S. Schlamminger, Metrologia, 2017, 54, 633.

38. M. Thomas, D. Ziane, P. Pinot, R. Karcher, A. Imanaliev, F. Pereira Dos Santos, S. Merlet, F. Piquemal, and P. Espel, Metrologia, 2017, 54, 468.

39. G. Bartl, P. Becker, B. Beckhoff, H. Bettin, E. Beyer, M. Borys, I. Busch, L. Cibik, G. D’Agostino, E. Darlatt, M. Di Luzio, K. Fujii, H. Fujimoto, K. Fujita, M. Kolbe, M. Krumrey, N. Kuramoto, E. Massa, M. Mecke, S. Mizushima, M. Müller, T. Narukawa, A. Nicolaus, A. Pramann, D. Rauch, O. Rienitz, C. P. Sasso, A. Stopic, R. Stosch, A.
Waseda, S. Wundrack, L. Zhang, and X. W. Zhang, Metrologia, 2017, 54, 693.

40. CCM Detailed Note on the Dissemination Process after the Redefinition of the Kilogram, https://www.bipm.org/utils/ common/pdf/CC/CCM/CCM_Note-on-dissemination-afterredefinition.pdf.

41. Consultative Committee for Mass and Relative Quantities, Mise en pratique for the redefinition of the kilogram in the SI, https://www.bipm.org/utils/en/pdf/si-mep/SI-App2-kilogram. pdf.

42. CODATA Internationally Recommended 2018 Values of the Fundamental Physical Constants, https://physics.nist.gov/ cuu/Constants/index.html.

43. N. Kuramoto, L. Zhang, S. Mizushima, K. Fujita, Y. Azuma, A. Kurokawa, and K. Fujii, IEEE Trans. Instrum. Meas., 2017, 66, 1267.

44. M. Gläser, "Comprehensive Mass Metrology", ed. M. Kochsiek and M. Gläser, 1999, Chap. 2, Wiley-VCH, Weinheim.

45. OIML R111-1, "Weights of Classes E1, E2, F1, F2, M1, M1-2, M2, M2-3 and M3, Part 1: Metrological and Technical Requirements", 2004, International Organization of Legal Metrology, Paris.

46. G. A. Shaw, J. Stirling, J. A. Kramar, A. Moses, P. Abbot, R. Steiner, A. Koffman, J. R. Pratt, and Z. Kubarych, Metrologia, 2016, 53, A86.

47. G. A. Shaw, J. Stirling, J. Kramar, P. Williams, M. Spidell, and R. Mirin, Metrologia, 2019, 56, 025002.

48. IUPAC Commission on Isotopic Abundances and Atomic Weights, Atomic Weights of the Elements 2019, https:// www.qmul.ac.uk/sbcs/iupac/AtWt/.

49. Consultative Committee for Amount of SubstanceMetrology in Chemistry and Biology, Mise en Pratique for the Definition of the Mole in the SI, https://www.bipm.org/ utils/en/pdf/si-mep/SI-App2-mole.pdf. 